LA GRANJA:

REVISTA DE

CIENCIAS DE LA VIDA

pISSN:1390-3799; eISSN:1390-8596

http:/ / doi.org/10.17163/lgr.n31.2020.04
Artículo científico / Scientific paper

ANALÍTICA DE CONTAMINANTES

\title{
VERIFICACIÓN DEL MÉTODO ANALÍTICO DE ESPECTROSCOPÍA DE ABSORCIÓN ATÓMICA CON HORNO DE GRAFITO PARA LA CUANTIFICACIÓN DE CADMIO EN ALMENDRA DE CACAO (Theobroma cacao)
}

\author{
VERIFICATION OF THE ATOMIC ABSORPTION SPECTROSCOPY WITH GRAPHITE \\ FURNACE ANALYTICAL METHOD FOR QUANTIFICATION OF CADMIUM IN \\ COCOA ALMONDS (Theobroma cacao)
}

\section{Salomé Araujo-Abad*1 ${ }^{\oplus}$, Wilson Tapia ${ }^{2}$ • y Adrián Villamarín-Ortiz ${ }^{3}$}

${ }^{1}$ Centro de Biotecnología. Universidad Nacional de Loja, 110111, Loja, Ecuador.

2 Departamento de Química. Universidad de las Américas, 170503, Quito, Ecuador.

${ }^{3}$ Departamento de Ciencias de la Salud. Universidad Técnica Particular de Loja, 1101608, Loja, Ecuador.

*Autor para correspondencia: lourdes.araujo@unl.edu.ec

\begin{abstract}
Resumen
El método de espectroscopía de absorción atómica (AA) de llama para la determinación de cadmio (Cd) en almendra de cacao (Theobroma cacao) utilizado por Agrocalidad es tóxico para el ser humano y el ambiente; por ello, se pretende utilizar el método de espectroscopia de absorción atómica con horno de grafito (GFAAS) por ser más confiable y seguro. Así, se realizó la verificación de cuatro parámetros de desempeño del método GFAAS para cuantificar Cd en almendra de cacao utilizando material de referencia certificado (MRC) y muestras provenientes de cuatro fincas (A, B, C, D) ubicadas en la zona cacaotera de Ecuador, cantón Flavio Alfaro, provincia de Manabí. Se realizó una prueba inter-laboratorios y finalmente se elaboró el protocolo (PEE/B/14). Sobre el MRC (Cód. 07206B y 07167A) se verificó: linealidad, precisión, veracidad e incertidumbre de acuerdo con la Guía Eurachem de Eurolab España y Morillas (2016), y con el estándar IRAM 35050 (2001) se encontró linealidad entre 0 y 8 ppb con $R^{2}=0,9988$; desviación estándar de 0,0005 y 0,0022 respectivamente; sesgo en 0,007 y porcentaje de recuperación de 109,75; la incertidumbre estándar de 0,00013 y 0,00082. El contenido de Cd en las muestras de la finca A con 0,54 ppm, las Fincas B-D con 0,26 ppm y 0,15 ppm en la finca C. En la prueba inter-laboratorios se estableció la misma concentración de cadmio para la muestra C3 y, de acuerdo con lo estipulado por la Unión Europea, el cacao de las cuatro fincas podría ser exportado sin restricciones.
\end{abstract}

Palabras clave: Cadmio, cacao, verificación, análisis, método 


\begin{abstract}
The flame atomic absorption (AA) spectroscopy method for the determination of cadmium (Cd) in cocoa almond (Theobroma cacao) used by Agrocalidad is toxic to humans and the environment, reason for which the atomic absorption spectroscopy method with graphite furnace (GFAAS) was used, because it is more reliable and safer. Thus, four performance parameters of GFAAS method were used to quantify $\mathrm{Cd}$ in cocoa almond, by using certified reference material (MRC) and samples from four farms (A, B, C, D) located in the most important cocoa area of Ecuador, Flavio Alfaro city, province of Manabi. An interlaboratory test was performed and finally a protocol (PEE/B/14) was developed. Using the MRC (Code 07806B and 07167) was verified: linearity, precision, veracity and uncertainty in accordance with international standards, Eurachem Guide of Eurolab Spain and Morillas (2016), and with the standard IRAM 35050 (2001) were found linearity between 0 and $8 \mathrm{ppb}$ with $R^{2}=0,9988$; standard deviation 0.0005 and 0.0022 , respectively; slant was 0.007 and the recovery percentage was 109.75; standard uncertainty was 0.00013 and 0.00082 . The content of Cd in samples from farm A was $0.54 \mathrm{ppm}, \mathrm{B}$ and D farms $0.26 \mathrm{ppm}$ and $0.15 \mathrm{ppm}$ in farm C. In the interlaboratory test, the same concentration of $\mathrm{Cd}$ was established for simple $\mathrm{C} 3$ and, in accordance with the stipulated by the European Union, cocoa from the four farms could be exported without restrictions.
\end{abstract}

Keywords: Cadmium, cocoa, verification, analysis, method.

Forma sugerida de citar: Araujo-Abad, L.S., Tapia, W. y Villamarín-Ortiz, A. (2020). Verificación del método analítico de espectroscopía de absorción atómica con horno de grafito para la cuantificación de cadmio en almendra de cacao (Theobroma cacao). La Granja: Revista de Ciencias de la Vida. Vol. 31(1):56-70. http://doi.org/10.17163/lgr.n31.2020.04.

IDs Orcid:

Lourdes Salomé Araujo Abad: https:/ / orcid.org/0000-0001-7387-0363

Wilson Fabián Tapia Hernández: https: / orcid.org/0000-0002-7010-6324

Marcos Adrián Villamarín Ortiz: https://orcid.org/0000-0003-2768-9162

La Granja: Revista de Ciencias de la Vida 31(1) 2020:56-70.

(C)2020, Universidad Politécnica Salesiana, Ecuador. 


\section{Introducción}

Los granos de cacao son las semillas del árbol de Theobroma cacao (nativo de la Región amazónica de América del Sur), que se consumen ampliamente en todo el mundo (Almeida y Valle, 2007). Es un cultivo neotropical, perenne importante que crece a $20^{\circ}$ norte y $20^{\circ}$ sur de la línea ecuatorial aproximadamente (Shavez Beg y col., 2017). Se cultiva a una altura de menos de $400 \mathrm{msnm}$; el rango óptimo de temperaturas oscila entre $22^{\text {circ }} \mathrm{C}$ y $30^{\circ} \mathrm{C}$, dependiendo de la variedad (Damatta y col., 2018), y la lluvia debe ser de al menos 1500 a $2500 \mathrm{~mm}$ de agua al año.

Las almendras son de color marrón y están cubiertas en el exterior por un mucílago blanco y dulce que es comestible (Waizel-Haiat y col., 2012), que se procesan para obtener licor de chocolate, cacao en polvo y manteca de cacao que son los principales ingredientes del chocolate y una amplia gama de productos como bebidas de cacao, helados, productos de panadería que otorgan un sabor característico y distintivo a sus productos derivados (Dasgupta y Klein, 2014). Los granos de cacao son la materia prima para la industria multimillonaria que produce chocolate y productos de confitería. La importancia económica de la industria del chocolate en el mercado mundial se ha revisado recientemente (Squicciarini y Swinnen, 2016) subiendo un 13\% desde 2010 hasta alcanzar los USD \$ 101 mil millones en 2015, con Suiza como el país con el índice de consumo más alto (Wickramasuriya y Dunwell, 2018).

Ecuador, por sus condiciones geográficas y su riqueza en recursos biológicos, es el productor por excelencia de cacao fino de aroma $(63 \%$ de la producción mundial) proveniente de la variedad nacional, cuyo sabor ha sido reconocido durante siglos en el mercado internacional. Este tipo de grano es utilizado en todos los chocolates refinados. Del total de la exportación ecuatoriana se estima que, un $75 \%$ es cacao fino de aroma, mientras que el restante $25 \%$ pertenece a otras variedades como el CCN51. Ecuador se posiciona como el país más competitivo de América Latina en este campo, seguido por Venezuela, Panamá y México, que son países que poco a poco han incrementado su participación en el mercado mundial (Anecacao, 2019).
Varios atributos de la calidad del grano, tanto físicos como químicos, son requeridos por los fabricantes, compradores de cacao y, organismos de control para alentar a la comunidad del cacao hacia una producción de mejor calidad (CAOBISCO/ECA/FCC, 2015). Estas características de calidad incluyen el sabor, pureza y salubridad (p. ej., libre de bacterias, infestación, alérgenos, micotoxinas, metales pesados y residuos de plaguicidas), características físicas (p. ej., consistencia, rendimiento de material comestible del grano, tamaño y uniformidad, contenido de cáscara, contenido de grasa y contenido de humedad) y las características de la manteca de cacao (p. ej., libre de ácidos grasos) (Dasgupta y Klein, 2014). Algunos de los atributos de la calidad de grano como el contenido total de grasa, acidez, fenoles totales, ácidos orgánicos, metales pesados, aminoácidos, cafeína, teobromina, $\mathrm{pH}$, azúcares, contenido de macro y micronutrientes, han sido considerados en la propuesta de índice de calidad del cacao (CQI) para algunos tipos de grano (Araujo y col., 2014).

Los metales pesados se definen como elementos con una densidad superior a $5 \mathrm{~g} / \mathrm{cm}^{3}$ (NavarroAviñó, Aguilar Alonso y López-Moya, 2007). El cobre, hierro, magnesio y cinc en bajas concentraciones son esenciales para los procesos bioquímicos y fisiológicos en las plantas, mientras que arsénico, cadmio y plomo no tienen un papel conocido en ellas (Ali, Khan y Anwar Sajad, 2013). La acumulación de metales pesados en las plantas se ve afectada por varios factores como el $\mathrm{pH}$, el contenido de materia orgánica y la textura del suelo, el genotipo de la planta y el contenido de metales pesados en el medio de crecimiento. América Latina posee los niveles más altos de metales pesados en los granos de cacao, especialmente de cadmio y plomo (Bertoldi y col., 2016), en comparación con otros productores en el mundo. La presencia de metales pesados en los granos de cacao representa una amenaza para los productores de cacao, ya que un alto contenido de metales pesados podría afectar la exportación de los granos.

$\mathrm{Al}$ chocolate se le ha atribuido el logro de una salud y desarrollo de seres humanos óptimos, debido a su alto contenido de flavonoides que son cruciales para reducir el riesgo o a su vez retrasar el desarrollo de enfermedades cardiovasculares, cáncer y otras relacionadas con la edad (Cooper y col., 
2007).

En 2014, la Unión Europea anunció, mediante el Reglamento (UE) No 488/2014, planes para implementar regulaciones acerca de los productos de chocolate y cacao que contienen niveles excesivos de cadmio (Cd), que entraron en vigor el 1 de enero de 2019 (Comission Regulation EU, 2014). El incumplimiento de las regulaciones tendría importantes consecuencias económicas y sociales para los países productores de cacao, como el Ecuador, a partir de la fecha de entrada en vigor de dicho reglamento. El Cd ha recibido atención en la última década debido a su importancia en la calidad/seguridad de los alimentos, y en la salud humana, ya que el consumo de alimentos con alto contenido de este metal pesado podría conducir a disfunción tubular renal, formación de cálculos renales, alteración del metabolismo del calcio y defectos esqueléticos, endocrinos, reproductivos y respiratorios (Järup y Akesson, 2009).

Las técnicas analíticas para la determinación de Cd se basan en el uso de reactivos que actúan sobre la digestión del grano y que son altamente peligrosas. Uno de los reactivos de uso frecuente es el Agua Regia $\left(\mathrm{HCl}: \mathrm{HNO}_{3}\right)$, que se somete a calor dando como resultado vapores irritantes y corrosivos; el protocolo utilizado corresponde a una adaptación del método oficial AOAC: 999.11 (AOAC, 2005).

Por ser un método analítico más eficaz y menos tóxico para el ser humano y el ambiente, en el presente trabajo se verificó el desempeño del método de espectroscopia de absorción atómica con horno de grafito (GFAAS) en la determinación de $\mathrm{Cd}$ en almendra de cacao (Theobroma cacao), mediante la determinación de linealidad, precisión y veracidad (Eurolab España y col., 2016) e incertidumbre (IRAM 35050, 2001) sobre material de referencia certificado, y se validó el protocolo analítico para la determinación de la concentración de $\mathrm{Cd}$ en muestras de almendra de cacao; adicionalmente, se realizó una prueba de comparación inter laboratorios.

\section{Materiales y Métodos}

Primera fase: se realizó la verificación del método analítico evaluando los parámetros de linealidad, precisión, veracidad e incertidumbre, a partir de material certificado MRC 07206B y 07167A.

Segunda fase: se recolectaron las muestras de cacao (mazorcas) por personal designado de AGROCALIDAD, en cuatro fincas de la zona cacaotera del país: Flavio Alfaro-Manabí, Ecuador, denominadas como A, B, C, D. Posteriormente, en los laboratorios de Bromatología y Microbiología de AGROCALIDAD se analizaron las muestras correspondientes para determinar su contenido en Cd, previa verificación de estado de calibración de los instrumentos y materiales volumétricos a utilizar.

Tercera fase: se elaboró el protocolo PEE/B/14 para la determinación de $\mathrm{Cd}$ en la almendra de cacao por GFAAS, que contiene el proceso de preparación de las muestras (mazorca de cacao) desde su recepción, secado, homogenización, digestión por microondas y posterior lectura en el espectrómetro con horno de grafito.

\subsection{Verificación del método analítico}

Para la verificación del método analítico se tomó como referencia a la Guía Eurachem de Eurolab España y col., (2016), y al estándar IRAM 35050, (2001), siguiendo el protocolo PEE/B/14.

\subsubsection{Linealidad}

Se elaboró una curva con 10 concentraciones a partir del estándar de Cd de 20 ppb. Las concentraciones utilizadas fueron de 0 a $18 \mathrm{ppb}$. En la curva se expresó en el eje de las " $x$ " la concentración teórica en $\mu g / L$ y en el eje de las " $y$ " la concentración calculada en $\mu g / L$. A partir de la curva resultante se escogió el rango lineal y se elaboró una nueva curva. En este intervalo lineal se midió por triplicado un blanco, seis diluciones diferentes del estándar de 20 ppb y finalmente los MRC 07206B y 07167A.

\subsubsection{Precisión}

Se realizaron quince lecturas de cada MRC 07206B y 07167A y se calculó la desviación estándar $(s)$ de cada material, usando la siguiente fórmula:

$$
s=\sqrt{\frac{1}{n-1} \sum_{k=1}^{n}(x-\bar{x})^{2}}
$$

Desviación estándar $(s)$

Donde: 
$n=$ número de mediciones totales del material de referencia.

$\bar{x}=$ promedio de las lecturas.

$x=$ cada medición realizada.

\subsubsection{Veracidad}

Se realizaron diez lecturas para MRC 07206B, diez lecturas para tres blancos digestados y diez lecturas para tres blancos adicionados con 2, 3 y 4 ppb de Cd. Además; también se realizaron diez lecturas sobre tres muestras analizadas, en primera instancia, por el método que normalmente usaba el laboratorio, en el que se emplea una digestión ácida con $\mathrm{Agua}$ Regia $\left(\mathrm{HCl}: \mathrm{HNO}_{3}\right)$, seguida de una lectura mediante espectroscopía de absorción atómica (AA) de llama $y$, en segunda instancia, por el método GFAAS verificado y el protocolo de análisis propuesto en esta investigación.

Con los datos obtenidos para el MRC 07206B se procedió a realizar los cálculos con las siguientes fórmulas proporcionadas por Eurolab España y col., (2016):

$$
b=\bar{x}-x_{\text {ref }}
$$

Sesgo

$$
b(\%)=\frac{\bar{x}-x_{r e f}}{x_{r e f}} \times 100
$$

Sesgo Relativo en Porcentaje

$$
R(\%)=\frac{\bar{x}}{x_{\text {ref }}} \times 100
$$

Recuperación relativa

Donde:

$\bar{x}=$ media de las lecturas del MRC 07206B.

$x_{r e f}=$ valor teórico que el certificado otorga al material de referencia.

Con los valores calculados se obtuvo una medida del sesgo tomando en cuenta los efectos del laboratorio comparado con los datos del sesgo del método.

Para el análisis de los datos obtenidos de las mediciones de blancos digestados y blancos adicionados, se usó la siguiente fórmula Eurolab España y col., (2016):

$$
R^{\prime}(\%)=\frac{\bar{x}^{\prime}-\bar{x}}{x_{\text {adición }}} \times 100
$$

Recuperación relativa de adiciones

Donde:

$\bar{x}^{\prime}=$ valor medio de los blancos adicionados.

$\bar{x}=$ valor medio de los blancos digestados.

$x_{\text {adición }}=$ valor de concentración añadida.

Finalmente, con los datos obtenidos de la lectura de muestras por el método normalmente usado en el laboratorio, el método GFAAS verificado y el protocolo de análisis propuesto, se realizaron los cálculos en base a las siguientes ecuaciones Eurolab España y col., (2016):

$$
b=\bar{x}-\bar{x}_{r e f}
$$

Sesgo

$$
b(\%)=\frac{\bar{x}-\bar{x}_{r e f}}{\bar{x}_{r e f}} \times 100
$$

Sesgo Relativo en Porcentaje

$$
R(\%)=\frac{\bar{x}}{\bar{x}_{\text {ref }}} \times 100
$$

Recuperación relativa

Donde:

$\bar{x}=$ media de las lecturas de las muestras por el método antiguo.

$\bar{x}_{r e f}=$ media de las lecturas de las muestras por el método nuevo propuesto.

Con los valores calculados se obtuvo la medida del sesgo, en función al método GFAAS verificado y el protocolo de análisis propuesto, para así demostrar su eficacia y sustituir al método normalmente utilizado por AGROCALIDAD.

\subsubsection{Incertidumbre}

Se realizaron 10 lecturas de la concentración del material de referencia y se calcularon los valores de incertidumbre con las siguientes fórmulas (IRAM 35050, 2001):

$$
\bar{I}=\frac{1}{n} \sum_{k=1}^{n} I_{k}
$$

Promedio (I) de las lecturas

Donde:

$n=$ número de mediciones del material de referencia.

$I_{k}=$ cada medición de material de referencia.

$$
S\left(I_{k}\right)=\sqrt{\frac{1}{n-1} \sum_{k=1}^{n}\left(I_{k}-\bar{I}\right)^{2}}
$$


Desviación estándar $\left(I_{k}\right)$

Donde:

$n=$ número de mediciones totales del material de referencia.

$\bar{I}=$ promedio de las lecturas.

$I_{k}=$ cada medición realizada .

$$
S(\bar{I})=\frac{S\left(I_{k}\right)}{\sqrt{n}}
$$

Incertidumbre estándar $S(I)$

Donde:

$S\left(I_{k}\right)=$ la desviación estándar.

$n=$ número de mediciones totales del material de referencia.

\subsection{Determinación de cadmio en almendra de cacao}

Con las muestras obtenidas de las Fincas A, B, C y D se procedió de acuerdo al protocolo elaborado (Procedimiento $\mathrm{PEE} / \mathrm{B} / 14$ ) a la determinación de $\mathrm{Cd}$ en almendras de cacao en el que se detalla:

1. Procesamiento de la muestra: Se secaron las almendras de cacao en la estufa a una temperatura de $150{ }^{\circ} \mathrm{C}$, por un período de 24 horas y se verificó que la cáscara se desprendía con facilidad, de lo contrario se continuó el secado por 12 o 24 horas adicionales y se realizó una nueva verificación antes de sacar el cacao de la estufa. El descascarillado se realizó en forma manual con las muestras calientes para que su desprendimiento se realizara con mayor facilidad. Para descascarillar el grano, se presionó con las yemas de los dedos o golpeando suavemente con un pistilo de mortero. Se procesaron aproximadamente $20 \mathrm{~g}$ de muestra en el molino, hasta obtener un producto homogéneo. Se guardó la muestra procesada en frascos de polipropileno con el código de la muestra.

2. Digestión: Se pesó con precisión $0.5 \mathrm{~g}$ de muestra seca en el envase de digestión y se sometió a digestión por microondas según a los parámetros indicados por el equipo.

3. Preparación de estándares: Se preparó $20 \mathrm{ml}$ de solución madre de $\mathrm{Cd}$ de 1000 ppb. Se tomó $2 \mathrm{ml}$ de estándar de $\mathrm{Cd}$ de $1000 \mu \mathrm{g} / \mathrm{ml}$ con pipeta volumétrica y se aforó a $20 \mathrm{ml}$ con ácido nítrico al $1 \%$. A partir de esta solución, el equipo de absorción atómica por horno de grafito realizó de forma automática las diluciones correspondientes (1-5 ppb).

4. Lectura de las muestras: Se midió la concentración de cada muestra codificada en el equipo, que fue programado para realizar lecturas por triplicado sobre una misma muestra y el resultado se expresó en partes por billón (ppb).

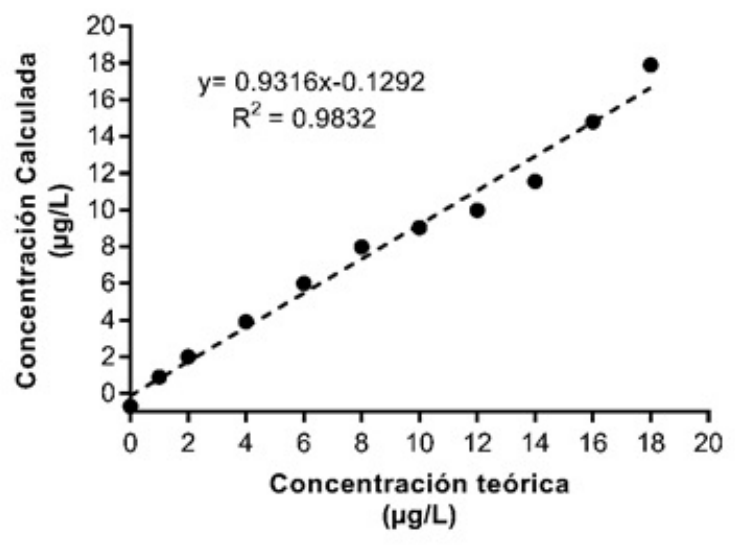

Figura 1. Rango lineal evaluado con diferentes concentraciones de estándar de Cd. 


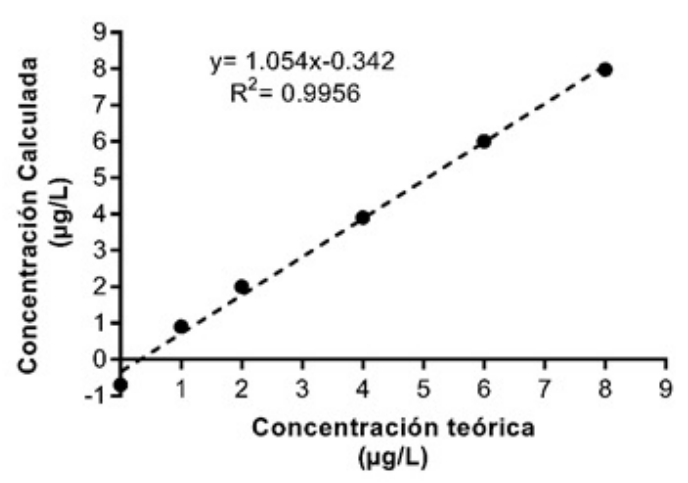

Figura 2. Rango lineal del método 0-8 ppb.

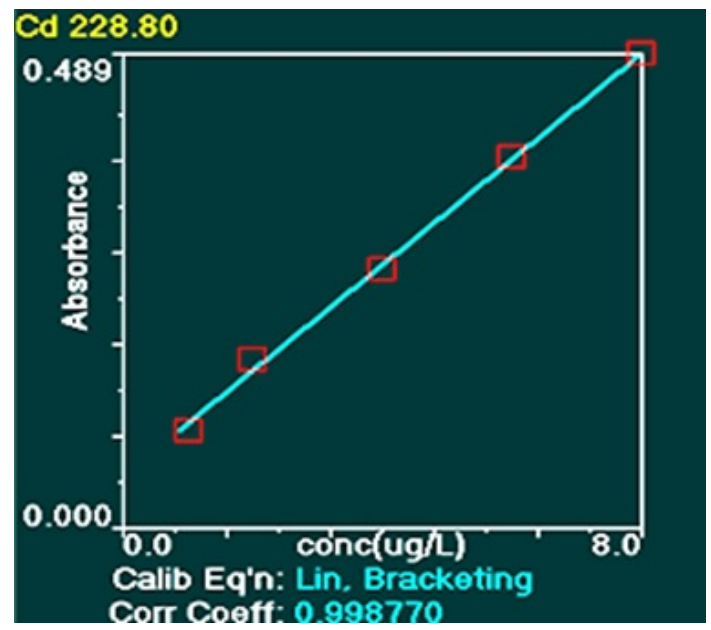

Figura 3. Rango lineal dado por el equipo.

\subsection{Análisis estadístico}

El análisis estadístico se realizó tomando en cuenta cada parámetro de verificación del método: linealidad, precisión, veracidad e incertidumbre, cada uno de los cuales proporciona fórmulas para el cálculo de medias, sesgo, sesgo relativo y recuperación. Además, en la determinación de Cd en almendras de cacao se realizó un análisis de varianza para determinar si existen diferencias significativas entre

En la Figura 1, se graficó la concentración teórica (0-18 ppb) frente a la proporcionada por el equipo (calculada) y, se procedió a realizar un análisis visual en el que se estableció que el rango lineal se encuentra entre 0 y 8 ppb (Figura 2 y 3 ). Se obser- las muestras obtenidas en cada finca. Se utilizó el programa estadístico Graphpad Prism 7.

\section{Resultados}

\subsection{Verificación del método analítico}

\subsubsection{Linealidad}

va que el rango lineal se encuentra entre 0 y $8 \mathrm{ppb}$ (Figura 2), con un coeficiente de residualidad de 0,9956, aceptable para procedimientos analíticos.

El método GFAAS arrojó una curva de calibra- 
ción (Figura 3), que presentó un coeficiente de residualidad de 0.998770 , mucho más exacta que la calculada, en razón que esta utiliza sólo los puntos de interés, eliminado errores de fondo. Para confirmar la linealidad del método y siguiendo el procedimiento de la Guía Eurachem (2016) se midió un blanco y seis disoluciones de concentraciones conocidas en el rango lineal dado por el equipo, que está representado en la Figura 4. En la Figura 4A. se observa que el coeficiente de residualidad fue de 0,9964; Figura 4B. 0,9976 y Figura 4C. 0,9952, que confirman la linealidad del método.
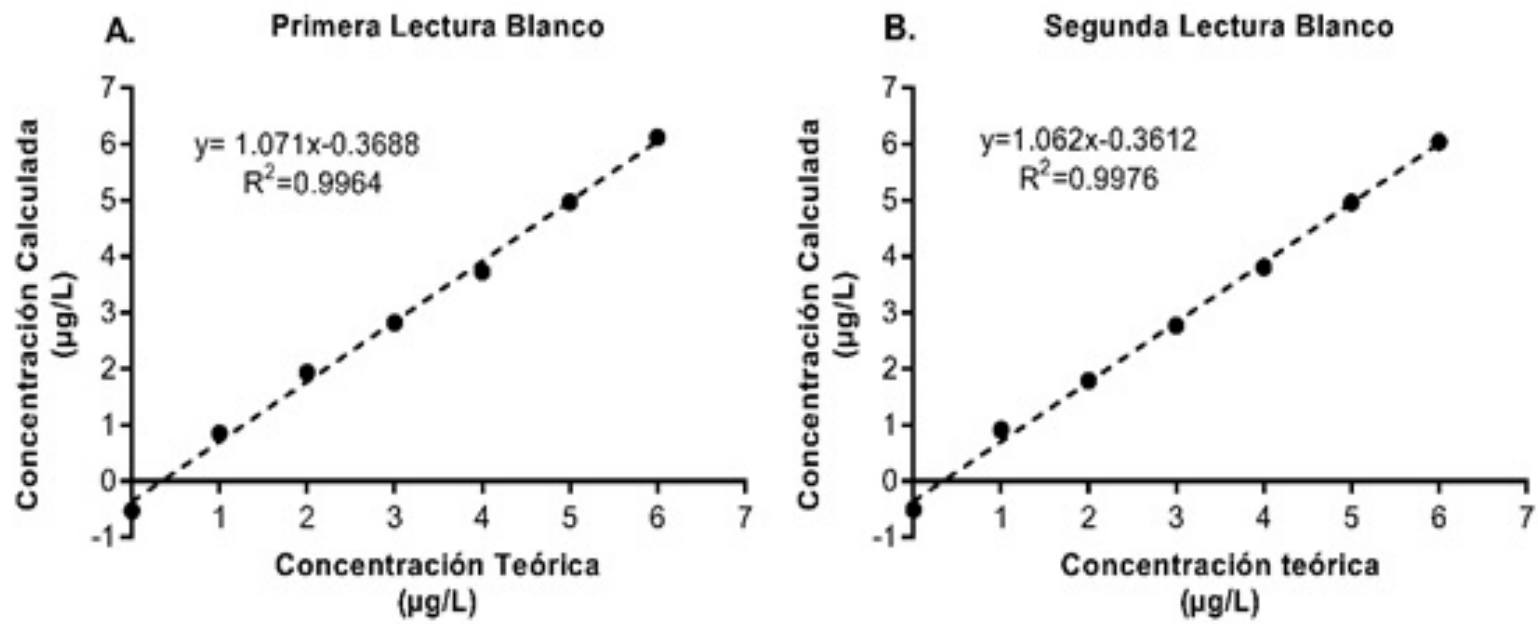

C. Tercera Lectura Blanco

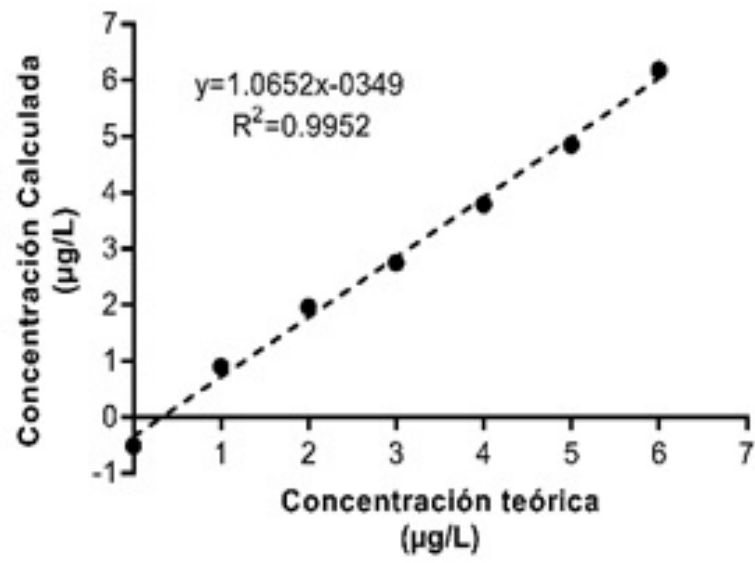

Figura 4. A. Primera lectura de un blanco y seis estándares de Cd; B. Segunda lectura de un blanco y seis estándares de Cd; C. Tercera lectura de un blanco y seis estándares de Cd.

\subsubsection{Precisión}

Se midió la precisión del analista, equipo y método analítico, mediante 15 lecturas de dos materiales de referencia certificados 07206B y 07167A, obteniéndose una desviación estándar mínima y menor a la dada por el fabricante del equipo que fue 0.02 como se observa en la Tabla 1. 
Tabla 1. Concentración de cadmio obtenida de 15 lecturas de dos materiales de referencia certificados de cacao en polvo.

\begin{tabular}{ccccc}
\hline \multirow{2}{*}{$\mathbf{N}^{\circ}$ de Lectura } & \multicolumn{4}{c}{ MATERIAL DE REFERENCIA CERTIFICADO } \\
\cline { 2 - 5 } & \multicolumn{2}{c}{$\begin{array}{c}\text { Lectura dada por el equipo } \\
\text { Concentración final calculada }\end{array}$} \\
\cline { 2 - 5 }$(\mu \mathrm{g} / \mathrm{L})$ & $\begin{array}{c}07167 \mathrm{~A} \\
(\mu \mathrm{g} / \mathrm{L})\end{array}$ & $\begin{array}{c}07206 \mathrm{~B} \\
(\mathrm{mg} / \mathrm{kg})\end{array}$ & $\begin{array}{c}07167 \mathrm{~A} \\
(\mathrm{mg} / \mathrm{kg})\end{array}$ \\
\hline $\mathbf{1}$ & 1,608 & 1,656 & 0,0803 & 0,0820 \\
\hline $\mathbf{2}$ & 1,605 & 1,794 & 0,0801 & 0,0888 \\
\hline $\mathbf{3}$ & 1,612 & 1,604 & 0,0805 & 0,0794 \\
\hline $\mathbf{4}$ & 1,610 & 1,626 & 0,0804 & 0,0805 \\
\hline $\mathbf{5}$ & 1,609 & 1,656 & 0,0803 & 0,0820 \\
\hline $\mathbf{6}$ & 1,632 & 1,614 & 0,0815 & 0,0799 \\
\hline $\mathbf{7}$ & 1,605 & 1,637 & 0,0801 & 0,0810 \\
\hline $\mathbf{8}$ & 1,615 & 1,644 & 0,0806 & 0,0814 \\
\hline $\mathbf{9}$ & 1,610 & 1,655 & 0,0804 & 0,0819 \\
\hline $\mathbf{1 0}$ & 1,603 & 1,645 & 0,0800 & 0,0814 \\
\hline $\mathbf{1 1}$ & 1,608 & 1,656 & 0,0803 & 0,0820 \\
\hline $\mathbf{1 2}$ & 1,603 & 1,702 & 0,0800 & 0,0842 \\
\hline $\mathbf{1 3}$ & 1,611 & 1,668 & 0,0804 & 0,0826 \\
\hline $\mathbf{1 4}$ & 1,622 & 1,644 & 0,0810 & 0,0814 \\
\hline $\mathbf{1 5}$ & 1,631 & 1,656 & 0,0814 & 0,0820 \\
\hline Desviación estándar $(\boldsymbol{s})$ & & 0,0005 & 0,0022 \\
\hline
\end{tabular}

Nota: Los valores están reportados en miligramos por kilogramo. La concentración final calculada se realizó en base a la lectura del equipo por el volumen de aforo y factor de dilución, dividido para el peso de la muestra por mil.

\subsubsection{Veracidad}

En la medición de la veracidad se realizaron diez lecturas del material de referencia certificado $07167 \mathrm{~A}$; en base a los datos obtenidos se calculó la media, el sesgo, sesgo relativo en porcentaje y la recuperación relativa, usando como valor referencial el dato proporcionado por el certificado de material de referencia, como se observa en la Tabla 2. Adicionalmente se realizaron diez lecturas de tres blancos y diez lecturas de los mismos blancos adicionados con 2, 3 y 4 ppb de $\mathrm{Cd}$, con lo que se

\subsubsection{Incertidumbre}

Se midió diez veces la concentración de $\mathrm{Cd}$ de dos materiales de referencia 07206B y 07167A, y se calculó la media, desviación estándar e incertidumbre estándar (Tabla 5). obtuvo la media y porcentaje de recuperación, como se observa en la Tabla 3.

Finalmente se realizaron diez lecturas de tres muestras por el método normalmente usado por el laboratorio (AA), por el método GFAAS verificado y el protocolo propuesto, como se observa en la Tabla 4. En la Tabla 4 se puede observar que el sesgo en la lectura de las tres muestras por los dos métodos es mínimo y el porcentaje de recuperación es elevado, lo que da confiabilidad al método GFAAS.

La incertidumbre calculada midiendo la concentración de Cd de los MRC (07206B y 07167A) es de 0,00013 y 0,00082 , respectivamente (Tabla 5.), lo que indica que las mediciones realizadas no estuvieron afectadas por errores sistemáticos en el proceso. 
Tabla 2. Concentración de cadmio obtenida de 10 lecturas del material de referencia certificado de cacao en polvo.

\begin{tabular}{ccc}
\hline \multirow{2}{*}{$\mathbf{N}^{\circ}$ Lectura } & \multicolumn{2}{c}{ Material de Referencia } \\
\cline { 2 - 3 } & $\begin{array}{c}07167 \mathrm{~A} \\
(\mu g / L)\end{array}$ & $\begin{array}{c}07167 \mathrm{~A} \\
(\mathrm{mg} / \mathrm{Kg})\end{array}$ \\
\hline $\mathbf{1}$ & 1,614 & 0,080 \\
\hline $\mathbf{2}$ & 1,637 & 0,081 \\
\hline $\mathbf{3}$ & 1,655 & 0,082 \\
\hline $\mathbf{4}$ & 1,655 & 0,082 \\
\hline $\mathbf{5}$ & 1,645 & 0,081 \\
\hline $\mathbf{6}$ & 1,656 & 0,082 \\
\hline $\mathbf{7}$ & 1,612 & 0,080 \\
\hline $\mathbf{8}$ & 1,668 & 0,083 \\
\hline $\mathbf{9}$ & 1,623 & 0,080 \\
\hline $\mathbf{1 0}$ & 1,644 & 0,081 \\
\hline Media & & 0,081 \\
\hline Valor Referencial & 0,074 \\
\hline Sesgo & 0,007 \\
\hline Sesgo Relativo en porcentaje & 9,75 \\
\hline Recuperación relativa & 109,75 \\
\hline
\end{tabular}

Nota: Los valores reportados por el quipo están en $\mu g / L$. La concentración final calculada $(m g / K g)$, se realiza en base a la lectura del equipo por el volumen de aforo y factor de dilución, dividido para el peso de la muestra por mil.

\subsection{Determinación de cadmio en almen- dras de cacao}

Se analizaron muestras provenientes de cuatro fincas de Flavio Alfaro- Manabí nombradas A, B, C y D (Tabla 6), colectándose 15 muestras de la finca A, 9 muestras de la finca B, 4 de la finca $C$ y 5 de la finca $\mathrm{D}$, de acuerdo a protocolos internos de Agrocalidad, estableciendo las siguientes hipótesis:

Después de realizar el análisis de varianza (Tabla 7) al ser $p<0,05$ se acepta la hipótesis alternativa, es decir, la concentración de $\mathrm{Cd}$ en las almendras de cacao de al menos una finca difiere de las demás. La Finca A que tiene como promedio $0,546 \mathrm{mg} / \mathrm{Kg}$ siendo el valor más alto respecto a las demás fincas, cabe mencionar que entre la Finca B y D no existen diferencias significativas ya que en las dos fincas la concentración de $\mathrm{Cd}$ en la almendra de cacao fue de $0,260 \mathrm{mg} / \mathrm{Kg}$. La Finca C presenta la concentración
Hipótesis nula: la concentración de Cd en las almendras de cacao de las cuatro fincas no es significativamente diferente.

Hipótesis alternativa: la concentración de Cd en las almendras de cacao de al menos una finca difiere significativamente de las demás.

más baja de $\mathrm{Cd}$ siendo 0,146 $\mathrm{mg} / \mathrm{Kg}$.

\subsection{Prueba inter-laboratorios}

Se envió la muestra con el código C3 a los Laboratorios UBA ubicados en la ciudad de Guayaquil, Ecuador. El resultado reportado fue de 0,11 ppm de $\mathrm{Cd}$, lo cual coincide con el resultado obtenido en Laboratorio de Bromatología y Microbiología de Agrocalidad. 
Tabla 3. Lectura de tres blancos y blancos adicionados con 2, 3 y 4 ppb de cadmio.

\begin{tabular}{ccccccc}
\hline \multirow{2}{*}{$\mathbf{N}^{\circ}$ Lectura } & \multicolumn{3}{c}{$\begin{array}{c}\text { BLANCOS } \\
(\mu \mathbf{g} / \mathbf{L})\end{array}$} & & \multicolumn{3}{c}{$\begin{array}{c}\text { BLANCOS ADICIONADOS } \\
(\mu \mathbf{g} / \mathbf{L})\end{array}$} \\
\cline { 2 - 7 } & $\mathbf{B 2 V}$ & $\mathbf{B 3 V}$ & $\mathbf{B 4 V}$ & $\mathbf{B A 2 V}$ & $\mathbf{B A 3 V}$ & $\mathbf{B A 4 V}$ \\
\hline $\mathbf{1}$ & $-0,5278$ & $-0,7799$ & $-0,4077$ & 2,098 & 3,115 & 3,780 \\
\hline $\mathbf{2}$ & $-0,5017$ & $-0,7791$ & $-0,3903$ & 2,084 & 3,185 & 3,898 \\
\hline $\mathbf{3}$ & $-0,5106$ & $-0,7442$ & $-0,4038$ & 1,962 & 3,247 & 4,165 \\
\hline $\mathbf{4}$ & $-0,5613$ & $-0,7247$ & $-0,4415$ & 1,939 & 3,025 & 4,036 \\
\hline $\mathbf{5}$ & $-0,4176$ & $-0,7153$ & $-0,4453$ & 1,962 & 3,004 & 4,054 \\
\hline $\mathbf{6}$ & $-0,5200$ & $-0,7298$ & $-0,4692$ & 1,963 & 3,027 & 4,076 \\
\hline $\mathbf{7}$ & $-0,6586$ & $-0,7062$ & $-0,4406$ & 1,876 & 3,057 & 4,185 \\
\hline $\mathbf{8}$ & $-0,5363$ & $-0,7423$ & $-0,4894$ & 1,878 & 2,947 & 4,196 \\
\hline $\mathbf{9}$ & $-0,5810$ & $-0,7847$ & $-0,4962$ & 1,890 & 3,030 & 4,223 \\
\hline $\mathbf{1 0}$ & $-0,5134$ & $-0,7700$ & $-0,4194$ & 1,800 & 3,137 & 4,263 \\
\hline Media & $-0,53283$ & $-0,74762$ & $-0,44034$ & 1,945 & 3,077 & 4,088 \\
\hline \multicolumn{7}{c}{ Recuperación $(\%)$} \\
\hline
\end{tabular}

Nota: Las unidades están reportadas en $(\mu g / L)$. El código B2V significa: Blanco número dos veracidad. BA2V significa, BA2: Blanco adicionado con 2 ppb de Cd; V: Veracidad.

Tabla 4. Concentración de cadmio en tres muestras evaluadas por GFAAS y AA.

\begin{tabular}{ccccccc}
\hline \multirow{2}{*}{ Lectura } & \multicolumn{2}{c}{ Lectura de muestras GFAAS } & \multicolumn{2}{c}{ Lectura de muestras AA } \\
\cline { 2 - 7 } & $\mathbf{A 8 A}$ & $\mathbf{B 2 3 A}$ & $\mathbf{D 6 A}$ & $\mathbf{A 8 A}$ & $\mathbf{B 2 3 A}$ & $\mathbf{D 6 A}$ \\
\hline $\mathbf{1}$ & 1,356 & 0,174 & 0,991 & 1,348 & 0,178 & 0,985 \\
\hline $\mathbf{2}$ & 1,348 & 0,177 & 0,996 & 1,387 & 0,174 & 0,981 \\
\hline $\mathbf{3}$ & 1,358 & 0,178 & 0,989 & 1,356 & 0,180 & 0,964 \\
\hline $\mathbf{4}$ & 1,363 & 0,175 & 0,983 & 1,364 & 0,173 & 0,994 \\
\hline $\mathbf{5}$ & 1,361 & 0,176 & 0,987 & 1,377 & 0,178 & 0,997 \\
\hline $\mathbf{6}$ & 1,371 & 0,174 & 0,981 & 1,374 & 0,180 & 0,999 \\
\hline $\mathbf{7}$ & 1,376 & 0,180 & 0,998 & 1,371 & 0,171 & 0,997 \\
\hline $\mathbf{8}$ & 1,368 & 0,182 & 0,993 & 1,350 & 0,178 & 0,986 \\
\hline $\mathbf{9}$ & 1,373 & 0,182 & 1,001 & 1,336 & 0,173 & 0,992 \\
\hline $\mathbf{1 0}$ & 1,366 & 0,180 & 0,989 & 1,342 & 0,173 & 0,988 \\
\hline Media & $\mathbf{1 , 3 6 4}$ & $\mathbf{0 , 1 7 8}$ & $\mathbf{0 , 9 9 1}$ & $\mathbf{1 , 3 6 1}$ & $\mathbf{0 , 1 7 6}$ & $\mathbf{0 , 9 8 8}$ \\
\hline Sesgo & 0,004 & 0,002 & 0,003 & & & \\
\hline Sesgo \% & 0,26 & 1,16 & 0,29 & & & \\
\hline R \% & 100,26 & 101,16 & 100,29 & & & \\
\hline
\end{tabular}

Nota: Las unidades están dadas en $m g / K g$. El código A8A significa: A: El nombre otorgado a la Finca; 8A: numeración de la muestra.

\section{Discusión}

La verificación del método analítico de espectroscopia de absorción atómica con horno de grafito en el Laboratorio de Bromatología y Microbiología de Agrocalidad, cumplió con los parámetros de desempeño: linealidad, precisión, veracidad de acuerdo a la Guía Eurachem (Eurolab España y col.,
2016) e incertidumbre de acuerdo con la Guía para el Cálculo de Incertidumbre del Instituto Argentino de Normalización y Certificación IRAM 35050, (2001). La linealidad se trabajó con una curva en un rango de 0 a $8 \mathrm{ppb}$, cuyo $R^{2}=0,998770$, que por su cercanía a +1 indica que existe una "perfecta" correlación entre las variables, siendo aceptable para mediciones analíticas (Gaddis y Gaddis, 1990). 
Tabla 5. Concentración de cadmio de dos materiales de referencia

\begin{tabular}{|c|c|c|c|c|}
\hline \multirow{4}{*}{$\mathbf{N}^{\circ}$ Lectura } & \multicolumn{4}{|c|}{ MATERIAL DE REFERENCIA CERTIFICADO } \\
\hline & \multicolumn{2}{|c|}{ Lectura dada por el equipo } & \multicolumn{2}{|c|}{ Concentración final calculada } \\
\hline & 07206B & 07167A & 07206B & 07167A \\
\hline & $(\mu g / L)$ & $(\mu g / L)$ & $(m g / K g)$ & $(m g / K g)$ \\
\hline 1 & 1,608 & 1,656 & 0,0803 & 0,0820 \\
\hline 2 & 1,605 & 1,794 & 0,0801 & 0,0888 \\
\hline 3 & 1,612 & 1,604 & 0,0805 & 0,0794 \\
\hline 4 & 1,610 & 1,626 & 0,0804 & 0,0805 \\
\hline 5 & 1,609 & 1,656 & 0,0803 & 0,0820 \\
\hline 6 & 1,632 & 1,614 & 0,0815 & 0,0799 \\
\hline 7 & 1,605 & 1,637 & 0,0801 & 0,0810 \\
\hline 8 & 1,615 & 1,644 & 0,0806 & 0,0814 \\
\hline 9 & 1,610 & 1,655 & 0,0804 & 0,0819 \\
\hline 10 & 1,603 & 1,645 & 0,0800 & 0,0814 \\
\hline \multicolumn{3}{|c|}{ Media } & 0,0804 & 0,0818 \\
\hline \multicolumn{3}{|c|}{ Desviación estándar } & 0,0004 & 0,0026 \\
\hline \multicolumn{3}{|c|}{ Incertidumbre estándar } & 0,00013 & 0,00082 \\
\hline
\end{tabular}

Nota: Los valores están dados en miligramos por kilogramo. La concentración final calculada se realiza en base a la lectura del equipo por el volumen de aforo y factor de dilución, dividido para el peso de la muestra por mil.

Tabla 6. Concentración de cadmio en almendra de cacao de las Fincas A, B, C y D.

\begin{tabular}{ccccc}
\hline \multirow{2}{*}{ Muestra } & \multicolumn{4}{c}{ Concentración de cadmio $(\mathbf{m g} / \mathbf{K g})$} \\
\cline { 2 - 5 } & Finca A & Finca B & Finca C & Finca D \\
\hline $\mathbf{1}$ & 0,60 & 0,60 & 0,12 & 0,12 \\
\hline $\mathbf{2}$ & 0,79 & 0,10 & 0,18 & 0,11 \\
\hline $\mathbf{3}$ & 0,60 & 0,01 & 0,11 & 0,24 \\
\hline $\mathbf{4}$ & 0,88 & 0,34 & 0,17 & 0,30 \\
\hline $\mathbf{5}$ & 0,74 & 0,30 & & 0,54 \\
\hline $\mathbf{6}$ & 0,89 & 0,40 & & \\
\hline $\mathbf{7}$ & 0,50 & 0,40 & & \\
\hline $\mathbf{8}$ & 0,54 & 0,09 & & \\
\hline $\mathbf{9}$ & 0,55 & 0,11 & & \\
\hline $\mathbf{1 0}$ & 0,49 & & & \\
\hline $\mathbf{1 1}$ & 0,54 & & & \\
\hline $\mathbf{1 2}$ & 0,15 & & & \\
\hline $\mathbf{1 3}$ & 0,13 & & & \\
\hline $\mathbf{1 4}$ & 0,40 & & & \\
\hline $\mathbf{1 5}$ & 0,39 & & \\
\hline
\end{tabular}

Nota: Los valores están expresados en miligramos por kilogramo. Los números de muestra no corresponden a la numeración que se otorgó a las muestras en el etiquetado

La precisión para dos materiales de referencia certificados (07206B y 07167A) arrojaron una desviación estándar de 0,0005 y 0,0022, respectivamente, lo cual implica que las mediciones realizadas por el analista fueron precisas y repetibles. En cuanto a la veracidad, se comprobó que la metodología propuesta arroja resultados similares a la metodología no estandarizada y tóxica que el Laboratorio de 
Tabla 7. Resumen del análisis de varianza

\begin{tabular}{lcccc}
\hline Grupos & Cuenta & Suma & Promedio & Varianza \\
\hline Finca A & 15 & 8,190 & 0,546 & 0,051 \\
\hline Finca B & 9 & 2,350 & 0,261 & 0,038 \\
\hline Finca C & 4 & 0,585 & 0,146 & 0,001 \\
\hline Finca D & 5 & 1,310 & 0,262 & 0,031 \\
\hline
\end{tabular}

Bromatología y Microbiología de Agrocalidad empleaba para los análisis de $\mathrm{Cd}$, puesto que el sesgo entre las dos metodologías fue mínimo y el porcentaje de recuperación fue mayor a $100 \%$ por lo que cumple con lo mencionado por Ospina y Zapata, (2012), quienes señalan que el porcentaje de recuperación en un método analítico debe ubicarse en un rango de $80 \%$ a $120 \%$. Además, es comparable con la validación de (Lo Dico y col., 2018) en la que obtienen un porcentaje de recuperación de $110 \%$. La incertidumbre estándar fue de 0,00013 y 0,00082, lo cual otorga confiabilidad al método propuesto y lleva a suponer que trabajar en un ambiente cerrado y con los demás equipos del laboratorio apagados conlleva a minimizar los errores tal como menciona (Gonzaga, 2016).

Una investigación relacionada es la de Acosta y Pozo, (2013) en la que se aplicó el método oficial AOAC 999.11, donde no se evidencia la verificación de los parámetros de desempeño en el laboratorio de Agrocalidad, por lo que sus resultados no son confiables. Sin embargo, la misma autora recomienda usar para la digestión de la muestra un tipo de digestión por microondas, la cual es más rápida y eficaz, por lo que en la presente investigación se usó dicha metodología de digestión basada en la norma (AOAC, 2010), obteniéndose buenos resultados.

En investigaciones relacionadas se usa en su mayoría la técnica de espectroscopía acoplada a llama con una digestión perclórica como la de Mite, Carrillo y Durango, (2010) y, una digestión nítrica previa seguida de la lectura por espectroscopia de emisión atómica acoplado a plasma (ICP-MS) de Chavez y col., (2015); sin embargo, se puede usar la ICP-MS después de una digestión por microondas, que otorga mayor especificidad del análisis (Lo Dico y col., 2018).

De acuerdo con lo estipulado por la Unión Europea ( $0,8 \mathrm{ppm}$ de $\mathrm{Cd}$ en cacao en polvo), que entró en vigor en enero de 2019, el cacao de las cuatro fincas presentadas en este estudio podría ser exportado sin restricciones, habiendo estado sus niveles (0.546 A; 0.261 B; 0.146 C; 0.262 D) dentro del rango permitido. De igual forma cumplen con lo indicado por el Comité del Codex sobre contaminantes en alimentos (CCCF), que, en la $9^{\circ}$ sesión celebrada en los Países Bajos, estipula un límite de 1,5 ppm (CCCF, 2015).

De acuerdo a Chavez y col., (2015) la diferencia en el contenido de $\mathrm{Cd}$ en las cuatro fincas se puede atribuir a la existencia de contaminación por metales pesados en el agua de riego o contaminación del suelo (Rankin y col., 2005), por otra parte Acosta y Pozo, (2013) hacen referencia a la ubicación de los cultivos, es decir, si se encuentran cerca de la carretera asfaltada, existe mayor probabilidad de acumular metales pesados tanto en el suelo como en las partes aéreas de las plantas por las emisiones de los vehículos circulantes a la atmósfera, lo que no ocurriría con las fincas que se encuentran alejadas de la carretera.

En adición a lo mencionado, Mite, Carrillo y Durango, (2010) acotan que la contaminación por Cd de las almendras de cacao puede darse además por la quema de residuos urbanos, el uso de lodos urbanos en la agricultura, agroquímicos y contaminación por derivados del petróleo al secar el cacao en carreteras. Por su parte, en una investigación reciente de Argüello y col., (2019) se hace referencia a que la concentración de Cd en la almendra de cacao puede variar de una finca a otra respecto al genotipo (CCN-51 vs. Nacional) cultivado.

A pesar de que este estudio no se centró en la determinación de $\mathrm{Cd}$ en almendras de caco sino en una validación del método analítico para cuantificar este metal, se ha logrado aportar significativamente al desarrollo de posteriores estudios en donde se use GFAAS y, se investigue la trazabilidad del Cd hasta llegar a las almendras, siendo evidente que el suelo es el mayor suministro de este metal, pudién- 
dose implementar estrategias de mitigación, como la biorremediación.

\section{Conclusiones}

Se verificaron con éxito los parámetros de desempeño del método analítico de espectroscopia de absorción atómica con horno de grafito, con lo cual se aseguró que el método es confiable y puede ser usado en el Laboratorio de Bromatología y Microbiología de Agrocalidad. El protocolo (PEE/B/14) elaborado permite la aplicación correcta del método en el análisis de $\mathrm{Cd}$ en la almendra de cacao puesto que define un procedimiento operativo estándar que asegura el fiel cumplimiento de los requisitos de ensayo para obtener resultados confiables.

Por otra parte, se evidenciaron diferencias en la concentración de $\mathrm{Cd}$ en la almendra de cacao de las fincas objeto de estudio que están relacionadas a la procedencia de las muestras. Sin embargo, todas las muestras analizadas revelaron concentraciones de Cd inferiores a lo estipulado por la Unión Europea ( 0,8 ppm de $\mathrm{Cd}$ en cacao en polvo) además, cumplen con lo indicado por el CCCF, un límite de 1,5 ppm, por lo que este cacao podría ser exportado sin restricciones.

La prueba inter-laboratorio que se llevó a cabo ratifica la validez del método verificado en esta investigación. En vista de la diferencia en la concentración de $\mathrm{Cd}$ de acuerdo con la procedencia de las muestras, es recomendable realizar otras investigaciones referentes a los factores que intervienen en la contaminación de este elemento en el cacao.

\section{Agradecimientos}

La presente investigación fue llevada a cabo en el Laboratorio de Bromatología y Microbiología de Agrocalidad, como parte del Proyecto Nacional del Cacao, Proyecto: I001 Agrocalidad "Implementación de un proceso de análisis optimizado para determinar la concentración de cadmio en almendras de cacao fresco, fermentado y seco".

\section{Referencias}

AOAC (2005). «Official Method 999.11: Determination of Lead, Cadmium, Copper, Iron, and Zinc in Foods.» En: Online: https:/ / bit.ly/2XyGfGp.

- (2010). «AOAC Official Method 999.10: Lead, Cadmium, Zinc, Cooper, and Iron in Foods». En: William Horwitz and George W Latimer (eds) Official methods of analysis of AOAC International. 18th edn. Gaithersburg, MD.:AOAC International, pp. 17-19. Online: https://bit.ly/325aDag.

Acosta, S. y P. Pozo (2013). «Determinación de cadmio en la almendra de cacao (Theobroma cacao) de cinco fincas ubicadas en la vía Santo Domingo - Esmeraldas, mediante espectrofotometría de absorción atómica con horno de grafito». En: infoANALÍTICA 1.1, Onine: https: / / bit.ly / 2S3GuDm.

Ali, H., E. Khan y M. Anwar Sajad (2013). «Phytoremediation of heavy metals-Concepts and applications». En: Chemosphere 91.7, 869-881. Online: doi: 10.1016/j.chemosphere.2013.01.075.

Almeida, A. A. y R. R. Valle (2007). «Ecophysiology of the cacao tree». En: Brazilian Journal of Plant Physiology 19.4, Sociedade Brasileira de Fisiologia Vegetal, 425-448. Online: https : / / bit.ly / 2SAb5KD.

Anecacao (2019). CACAO NACIONAL, Un producto emblemático del Ecuador. URL: https: / / bit.ly / 2XXpPGQ.

Araujo, Q. y col. (2014). «Cocoa Quality Index - A proposal». En: Food Control 46, Elsevier Science, 49-54. Online: https:/ / bit.ly/2SBvpvm.

Argüello, D. y col. (2019). «Soil properties and agronomic factors affecting cadmium concentrations in cacao beans: A nationwide survey in Ecuador». En: Science of The Total Environment 649, 120-127. Online: https:/ / bit.ly/37F7vmF.

Bertoldi, D. y col. (2016). «Multielemental fingerprinting and geographic traceability of Theobroma cacao beans and cocoa products». En: Food Control 65, Elsevier Science, 46-53. Online: https: //bit.ly/3bHPAiC.

CAOBISCO/ECA/FCC (2015). Cocoa Beans: Chocolate and Cocoa Industry Quality Requirements. Ed. por M. J. End y R. Dand. Online: https:/ /bit.ly/ 2xB2PP9.

CCCF, ed. (2015). Programa Conjunto FAO/OMS sobre normas alimentarias. Documento de debate para el establecimiento de niveles máximos de cadmio en chocolate y productos derivados del cacao, pág. 66. 
Chavez, E. y col. (2015). «Concentration of cadmium in cacao beans and its relationship with soil cadmium in southern Ecuador». En: Science of the Total Environment 533, 205-214. Online: https:/ / bit. ly/38G1mYU.

Cooper, K. A. y col. (2007). «Cocoa and health: a decade of research». En: British Journal of Nutrition 99.1, 1-11. Online: https://bit.ly/38MixId.

Damatta, F. M. y col. (2018). «Physiological and agronomic performance of the coffee crop in the context of climate change and global warming: a Review». En: Journal of Agricultural and Food Chemistry 66.21, American Chemical Society, 5264-5274. Online: https : / / bit . ly / 2HLbUKx.

Dasgupta, A. y K. Klein (2014). «Tea, Coffee, and Chocolate: Rich Sources of Antioxidants». En: Antioxidants in Food, Vitamins and Supplements. Elsevier, 237-257. Online: https : / / bit . ly / 2SLHwWu.

Eurolab España y col. (2016). Guia Eurachem: La Adecuación al Uso de los Métodos Analíticos Una Guía de Laboratorio para Validación de Métodos y Temas Relacionados. 1 ed. Inf. téc. Eurachem, Online: https://bit.ly/2YDyhIy.

Gaddis, M. L. y G. M. Gaddis (1990). «Introduction to biostatistics: Part 6, Correlation and regression». En: Annals of emergency medicine 19.12, 1462-1468. Online: https:/ / bit.ly/2HIZFhx.

Gonzaga, P. A. (2016). «Validación de métodos analíticos para la determinación de metales Pesados, mediante absorción atómica en las matrices de aguas residuales, naturales y de consumo humano en los laboratorios de la UTPL». Trabajo de titulación. Universidad Técnica Particular de Loja. Online: https://bit.ly/2G1q09W.

IRAM 35050 (2001). Procedimientos para la evaluación de la incertidumbre de la medición. Inf. téc. Argentina: Instituto Argentino de Normalización. Online: https:/ / bit.ly/2XRnbma.

Järup, L. y A. Akesson (2009). "Current status of cadmium as an environmental health problem». En: Toxicol Appl Pharmacol 238.3, 201-208. Online: https://bit.ly/2SKVCaQ.
Lo Dico, G. M. y col. (2018). «Toxic metal levels in cocoa powder and chocolate by ICP-MS method after microwave-assisted digestion». En: Food Chemistry 245, 1163-1168. Online: https: / / bit . ly $/ 2 \mathrm{HHJfpN}$.

Mite, F., M. Carrillo y W. Durango (2010). «Avances del monitoreo de presencia de Cadmio en almendras de cacao, suelos y aguas en Ecuador». En: XII Congreso Ecuatoriano de la Ciencia del Suelo. Vol. 1, 1-21. Online: https:/ / bit.ly/2LgnFMt.

Navarro-Aviñó, J., I. Aguilar Alonso y J. LópezMoya (2007). «Aspectos bioquímicos y genéticos de la tolerancia y acumulación de metales pesados en plantas». En: Asociación Española de Ecología Terrestre 12.2, 10-25. Online: https: / / bit.ly / 2 NptmI7.

Ospina, C. y M. Zapata (2012). «Validación de la metodología de detección de los metales cadmio, níquel y plomo en agua tratada por absorción atómica de llama en el Laboratorio de Análisis de Aguas y Alimentos de la Universidad Tecnológica de Pereira». Trabajo de Grado. Universidad Tecnológica de Pereira. Online: https: / / bit. ly/2S62U7e.

Rankin, C. W. y col. (2005). «Lead contamination in cocoa and cocoa products: isotopic evidence of global contamination». En: Environmental health perspectives 113.10, National Institute of Environmental Health Science, 1344-1348. Online: https: //bit.ly/2HI5QlM.

Shavez Beg, M. y col. (2017). «Status, supply chain and processing of Cocoa - A review». En: Trends in Food Science \& Technology 66, 108-116. Online: https://bit.ly/2SLObAc.

Squicciarini, M. P. y J. F. M. Swinnen (2016). The Economics of Chocolate. Oxford University Press.

Waizel-Haiat, S. y col. (2012). «Cacao y chocolate: seducción y terapéutica». En: Anales Médicos 57.3, 236-245. Online: https: / bit.ly /2LNJ13t.

Wickramasuriya, A. M. y J. M. Dunwell (2018). «Cacao biotechnology: current status and future prospects». En: Plant Biotechnol Journal 16.1, 4-17. Online: https:/ / bit.ly/3c02qZw. 